\title{
Development of an Innovative Mobile Phone- Based Newborn Care Training Application
}

\author{
Sherri Bucher, Ph.D., MA ${ }^{1}$, Elizabeth Meyers ${ }^{2}$, Bhavani Singh \\ Agnikula Kshatriya ${ }^{2}$, Prem Chand Avanigadda ${ }^{3}$, and Saptarshi \\ Purkayastha, Ph.D. ${ }^{2}$ \\ Indiana University School of Medicine. Dept of Pediatrics, Indianapolis, USA. \\ ${ }^{2}$ School of Informatics \& Computing, IUPUI. Indianapolis, USA. \\ ${ }^{3}$ Computer \& Information Science, IUPUI. Indianapolis, USA.
}

\begin{abstract}
Mobile infrastructure in low- and middle-income countries (LMIC) has shown immense potential to reach the unreachable. Healthcare providers (HCP) are one such group who are at the frontline of the fight against infant mortality in LMICs. Mortality among newborn infants (birth to 28 days) now accounts for around $45 \%$ of all under 5years child mortality. Birth asphyxia is one of the three leading causes of newborn death; neonatal resuscitation training, among health care providers, reduces mortality from birth asphyxia. We have developed a mobile phone-based training app, called mobile Helping Babies Survive (mHBS), to support the training of health care providers on neonatal resuscitation. mHBS is integrated with the District Health Information System (DHIS2) platform, which is used in over 60 countries around the world. The mHBS/DHIS2 training app is a part of an application suite which includes another DHIS2-linked data collection app, mHBS tracker. The mHBS training application has the potential to scale-up integration with other neonatal training apps. Ultimately, the mHBS training suite will provide new insights into healthcare worker education along with the necessary tools for effective care of newborn babies.
\end{abstract}

Keywords: eLearning, mHealth, mHBS, DHIS2, Healthcare providers, Newborn care, SDG.

\section{Background}

Life-threatening realities for infants in Low- and Middle-Income Countries (LMICs) include birth asphyxia, infections, and birth trauma, contributing to 2.6 million annual preventable neonatal deaths occurring in the first month of an infant's life [1][2]. Early recognition of neonatal complications, management of care, and delivery of interventions by skilled healthcare providers (HCPs) are critical components for reduction of global rates of infant mortality [3].

In the past few years, there has been recognition that mortality which occurs during the neonatal period - birth to 28 days of life among liveborn infants underlies a large proportion of all infant and under 5-years child mortality. Low/middle-income countries bear a disproportionate burden for newborn death. Accordingly, global health partners, such as the American Academy of Pediatrics have made significant progress in formalizing and disseminating evidence-based guidelines for newborn care in low/middle-income countries. The flagship program of the Helping Babies Survive suite of newborn care interventions, Helping Babies Breathe (HBB), has been implemented in 80 countries and reached more than 300,000 birth attendants [4][5][6]. However, program implementation barriers exist. These include a lack of quality assurance for initial and refresher trainings [7], limited access to training materials and resources, which are currently scattered across various partner websites, [8], and knowledge gaps regarding engagement metrics on selfmotivated learning.

At the same time, mobile penetration rates in LMICs amount to $70 \%$ of the world's mobile phone subscriptions [9]. There is increasing accessibility to mobile device ownership and access bolstered by reduced pricing for

This is the author's manuscript of the article published in final edited form as:

Bucher, S., Meyers, E., Kshatriya, B. S. A., Avanigadda, P. C., \& Purkayastha, S. (2019). Development of an Innovative Mobile Phone-Based Newborn Care Training Application. In A. Abraham, N. Gandhi, \& M. Pant (Eds.), Innovations in Bio-Inspired Computing and Applications (pp. 361-374). Springer International Publishing. https://doi.org/10.1007/978-3-030-16681-6_36 
smartphones and tablets, improved connectivity, and enabling technologies such as mobile banking and social platforms, which encourage previously disconnected populations to acquire mobile phones and tablets and engage with these devices more frequently [10][11]. In accordance with this changing ecosystem, a plethora of mobile health (mHealth) and electronic learning (eLearning) applications have been developed to address the pernicious issue of maternal and neonatal mortality [12]. Furthermore, data analysis and health management information systems such as the open-source District Health Information System (DHIS2) [13], which is already used in over sixty countries [14], have created a platform to support data collection specifically tailored to build support health programs. Steadily, use of the DHIS2 platform is improving health system capacity for the delivery of evidence-based health interventions, and monitoring and evaluation of healthcare programs, in LMICs. At this opportune time, our team has developed the Mobile Helping Babies Survive (mHBS) app to support healthcare workers as they obtain facilitated training for neonatal resuscitation, and maintain their knowledge, skills, and competencies over time. Through the integration of mHBS training and mHBS tracker app with DHIS2 [15], we link our training and data collection suite with an already scaled system for health information within LMICs.

This strategy supports the potential for scale-up and sustainability of mHBS. In our development efforts, we employ agile development, best practices from eLearning, and strategies from user-centered design. Furthermore, our efforts adhere to the Principles for Digital Development [16]. We have created an opensource, Android-based mobile training application called mHBS [17]

\section{Methodology}

The mHBS training application followed human-centered design to maximize adoption potential and create a simple experience for users across all technical skill levels. First, the technology team, consisting of 7 members, engaged in an extensive literature review regarding existing mHealth applications to understand the burdens faced by nurses, skilled birth attendants (SBAs), community and facility-based health care workers, and midwives in low- and middle-income countries (LMICs). Then, four subsequent phases of design, programming, beta-evaluation, and re-design occurred. Throughout the development cycle, Iterative, agile development processes were employed, GitHub was used for documentation and collaboration to align with the opensource doctrines. Weekly scrum meetings were held for monitoring progress and addressing program bottlenecks.

\subsection{Design Phase}

Three members of the technology team participated in the design phase, which lasted for 3 months. This phase built upon two previous iterations of the mHBB application [17] that were developed by two previous teams, each of which used a different technology stack, and did not have integration with DHIS2. Our current application is based on the rationale that low-and-middle-income countries (LMICs) have made substantial investments in deploying the DHIS2 infrastructure where human resources in health system already use DHIS2 for routine data collection and program management. By utilizing user-centered design processes, and linking mHBS with DHIS2, we support each of the nine Principles for Digital Development.

Upon completion of the comprehensive literature review, and during the design phase, meetings were held with domain experts in the fields of Pediatrics and Bio-Health Informatics to further inform the technology team of the health system landscape, potential use cases, and to discuss technology constraints related to app development for low resourced settings [18]. A convergence of ideas led to the identification of core features, including the need to establish a link between the existing mHBS tracker and mHBS training app, utilizing the 
tracker app as a central point of entry, as a proof of concept for creating a seamless, linked suite of digital tools. Identification of this link as a critical component is due to its potential in extending the tracking of a healthcare provider (HCP) who is already registered in the mHBS tracker/DHIS2. This linkage also allows a mechanism for any number of custom mobile apps in this problem space to be modularly attached to the same portal. A key innovation of this approach is that, traditionally, DHIS2 has been used to monitor health service delivery by a facility or track entities that receive care at the facility. mHBS innovates by using entities at the level of individual healthcare provider.

In addition, it was decided that, to facilitate the user experience, the two apps should appear homogeneous, in terms of the interface, as a user toggles between them. This purposeful design of a seamless transition was expected to decrease the learning curve for a new user, as the style across applications would be more familiar. Therefore, design choices included the use of Google's Material design and a hamburger menu, along with other elements already present in the mHBS tracker. Educational content for the mHBS training app constituted the second key app deliverable, which included standardized videos and PDFs from global health partners, and sequential learning modules (courses). The videos were gathered from partner websites and tested for ideal compression, which led to WebM as the chosen file format.

An added restriction on educational content was that it must be stored and retrieved through a DHIS2 web API to allow full integration with the existing system. The mHBS training app was also designed for extensibility, to accommodate potential future resources, such as images. Other desired features included the need for the off-line mode to support intermittent $\mathrm{WiFi}$, which is prevalent in LMICs [19], along with the ability to capture user-resource interaction metrics (including how often a user viewed content, and for what length of time).

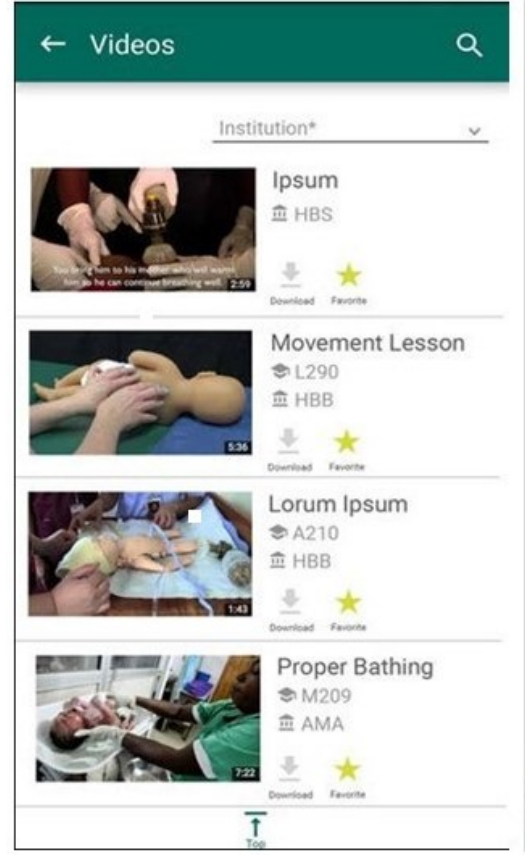

Fig. 1. Initial prototype showing video in a reusable list

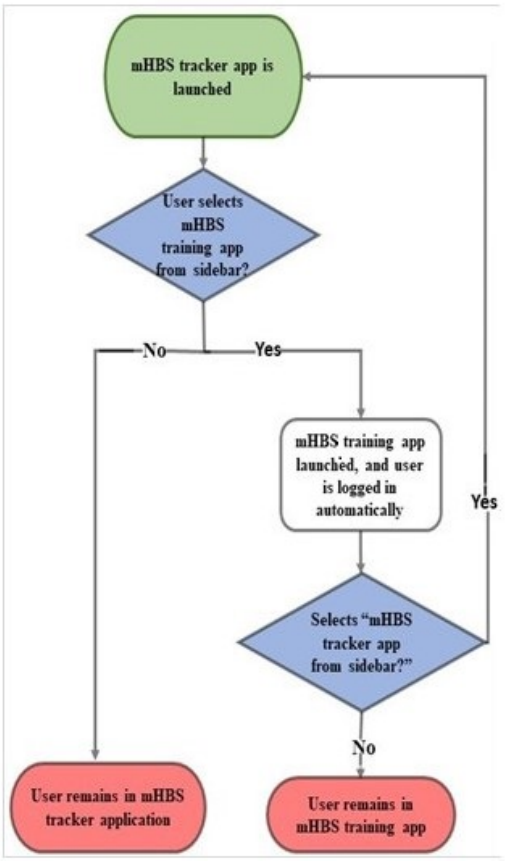

Fig. 2. The Process flow for linking with DHIS2 login

Following feature identification, user experience tools Balsamiq [20] and Marvel [21] were used to making two prototypes which were presented to the project team for re-evaluation. Finally, a high-fidelity prototype (Fig. 1) was created that synthesized features of the original mockups and development using Android Studio followed.

\subsection{Programming Phase}

\subsubsection{Dhis2 SDK}


Four members from the technology team participated in the programming phase, which lasted for ten months, and resulted in a deployable Android mobile app. Once the basic skeleton for the training app was created using the DHIS2 Software Development Kit (SDK), tasks were prioritized, (2) functionality improvements, and (3) appearance to support beta-test release. Our open-source project can be found at https://github.com/iupui-soic/mHBS-Tracker.

\subsubsection{Plugins}

We used com.github.barteksc:android-pdf-viewer:3.0.0-beta.5 and fabric.io plugins to display the PDF documents in the training app, send crashes and events to fabric server.

\subsubsection{Offline and online modes}

DHIS2 SDK doesn't have any existing API to support retrieving resources such as videos and PDFs that are stored in the DHIS2 platform. Health systems use the DHIS2 resource feature to share documents related to health policies, documentation, and other types of related content. But in our project, we utilize the DHIS2 resource feature to store training videos, images and PDF documents that can be used by the Healthcare providers (HCPs) for the self-directed, individually paced learning of knowledge, skills, and competencies in newborn care content. As for the database structure on the web server, a file or URL resource is stored under the single field "document," which supports multiple resource types. Each document contains information such as a tag, title, content type, URL, file permission status, and last update date, all of which is stored in Extensible Markup Language (XML) format. Even though DHIS2's primary usage is like a Data warehouse, it provides a general- purpose RESTful API for communication from external applications. Both the tracker and training app, as well as the DHIS2 Android SDK, uses the DHIS2 REST API for communication. The DHIS2 stored resources are available at the document's endpoint.

A collection of documents (/api/documents) returns an XML or JSON array, and depending on the Accept HTTP Header, a list of tags and titles which links to each document. Since many types of content (PDFs and Videos) can be stored together, the documents array needed to be parsed based on their content types. To handle this, an asynchronous background task was created to access the DHIS2 Web API and implement an Android XML Pull Parser interface to obtain relevant XML tags. The Pull Parser was used to iterate through the documents collection and gather document IDs and tags. An ID was then added to the URL path (api/documents/ID) to retrieve the content type and URL related to every document stored in DHIS2 resources.

\subsubsection{Integrating internal and external applications/resources}

The mobile Helping Babies Survive (mHBS) training app needed to have integration with internal applications such as mHBS tracker app (data collection and reporting app) and mHBS Aggregate app (data visualization), to switch among the applications seamlessly. The mHBS training app also needed to have integration with external applications supportive of maternal and newborn care program implementation, such as a Safe Delivery app [22], REDCap Mobile [23], Essential Care for Small Babies, and external resources such as DHIS2, to retrieve the training material.

The first integration activities involved linking the mHBS training and tracker apps. While the training app can be launched quickly from the tracker app, the user session must be preserved between the two separate applications. To accommodate this, a BroadcastReceiver [24] was implemented.

A broadcast is sent to the tracker app whenever the training app is launched, which allows the receiving of an intent that contains the username, password, and DHIS2 URL of the HCP from the tracker application. DHIS2 login is handled by the DHIS2 SDK through private, unmodifiable functions. Therefore, a custom login was created to logging in through the app to DHIS2 (fig. 2, 3-4).

Once the content types are retrieved, the documents are parsed into groups. Using the same background task, video thumbnails are gathered as bitmaps using a MediaMetadataRetriever [25]. Finally, content is downloaded to folders 
on the device using a DownloadManager [26]. The downloaded content is then displayed as part of an updated dynamic list similar to the prototype mockup shown in Figure 1. Users can click on the download icon and download the resources for offline viewing or "star" the content so that these can be marked as favorite. Prior to the beta-test release, "work in progress" code was disabled. Notably, this included course functionality and certain PDFs.

For purposes of the proof-of-concept, the Helping Babies Breathe (HBB) Second Edition neonatal resuscitation training guide for Providers Guide was deliberately left as the only visible PDF on the application, as it was of primary interest to gain feedback on font size and navigation within a single PDF, rather than displaying a breadth of options. The HBB Provider Guide was also identified as a critical resource that must always be available to a user.

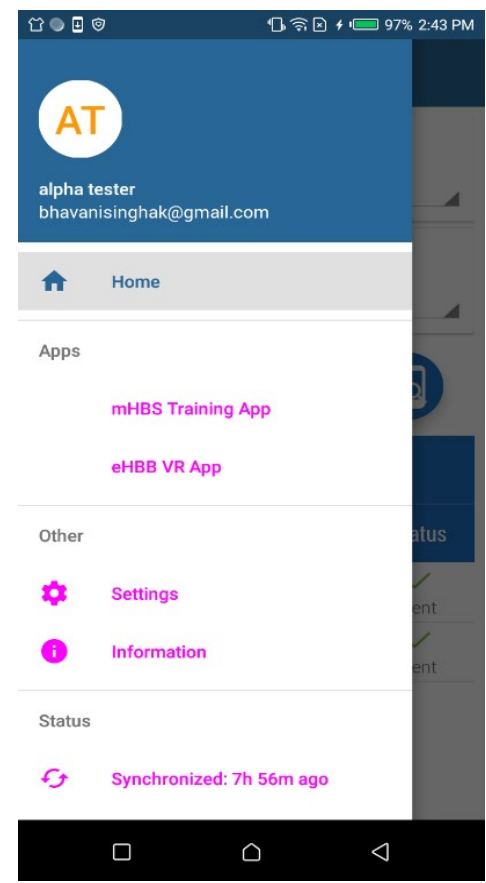

Fig. 3. mHBS tracker app showing a link

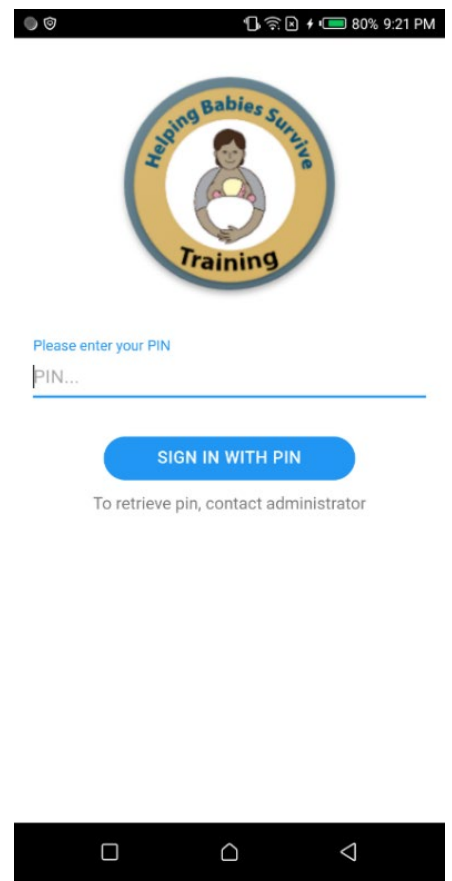

Fig. 4. mHBS training app

\subsubsection{Privacy and Security}

Initially, to address the privacy and security concerns of the training app users, the code was written to allow launching of the training app only from within the mHBS tracker app. Specific user credentials containing username and password were provided, allowing HCPs to launch the mHBS tracker securely.

\subsection{Beta Testing Phase}

The mHBS training app was released alongside the mHBS tracker for beta testing on Google Play Store as part of a public beta on March 26, 2018. The app was downloaded over 200 times during this phase, which lasted until May, 20, 2018. A user guide for beta testers was distributed, which included screenshots and instructions for app use, as well as a structured survey to capture the System Usability Scale (SUS) [24]. A more elaborate semi-structured feedback questionnaire was subsequently created in PowerPoint for qualitative usability testing of the mHBS Trainer and Tracker apps. This questionnaire was sent to our beta testers, which included global health partners, clinicians, and international neonatal resuscitation training experts. End-user feedback from the beta testers was discussed in our weekly scrum meetings.

The user feedback was generally positive, and included recommendations to increase the number of links between deeply nested pages to allow for more navigational control, along with improvements to app appearance through the inclusion of professional, uniform images, and implementing additional privacy and security features. 


\section{Results}

\subsection{Development}

\subsubsection{Cordova}

Based on user recommendations from the beta-testing phase, we removed DHIS2 SDK as underlying architecture for the mHBS training app and implemented a new mobile app development framework. This decision was in response to the fact that DHIS2 SDK has constraints which preclude our ability to efficiently and effectively modify the training app to address the suggestions. We selected Apache Cordova as a new platform for development to replace the DHIS2 SDK. Cordova is an open-source mobile development framework used to develop cross-platform mobile applications using HTML, CSS3, and JavaScript. We also used Framework7, which is a hybrid applications development HTML framework, to develop the mHBS Trainer app, which finally improved user interaction and experience.

\subsubsection{Plugins}

Along with the implementation of Cordova, we used eight new plugins to address the beta-testing recommendations. cordova-plugin-whitelist: For security and configurability of the training app by implementing a whitelist policy for navigating the application WebView. com-darryn-campbellcordova-plugin-intent: For sending and receiving of intents. cordova-pluginnetwork-information: For gaining information on the device's internet connection. cordova-plugin-dialogs: For getting native dialog user interface elements in the training app. cordova-plugin-secure-storage: For storing confidential credentials such as username, passwords, tokens, certificates, etc. cordova-fabric-plugin: For sending fabric events and crash analytics data to the fabric server. cordova-custom-config: For maintaining all plugins' version to be limited to initial Cordova application version. com.lampa.startapp: For launching integrated applications from the current training app.

\subsubsection{Offline and online modes}

Due to the implementation of cordova-plugin-network-information, it is now easy to verify if an HCP's device is offline or online. This further lead to coding the training app to collect and store app usage data as local application data whenever the app is used in offline mode, and sending of those data to DHIS2 server whenever a cellular or WiFi connection is activated.

\subsubsection{Integrating internal and external applications/resources}

Two plugins were implemented for the integration of internal applications, i.e., mHBS training app and mHBS tracker app. We have used com-darryn campbell-cordova-plugin-intent to receive intent from mHBS tracker which contains the username, password, server name, and pin numbers to access mHBS training. The intent also contains information about healthcare provider organization units and their id's (known as tracked entity instance id) for pushing training app usage data to the DHIS2 server. com.lampa.startapp plugin implementation leads to scaling up of training app integration with additional external newborn care applications. This feature also allows us to include links to resources of external partners; for the demonstration version of mHBS Trainer, we have included links to the American Academy of Pediatrics (Helping Babies Survive page) and PATH (Reprocessing Guidelines for Basic Neonatal Resuscitation Equipment in Resource-Limited Settings), as shown in Figure 5. This feature provides HCPs and other end-users and partners with ease of access to a wide variety of partner resources by which to strengthen knowledge at both the individual (self-directed learning) and institutional (program) levels.

\subsubsection{Privacy and security}

Vital features were added to the training app to address privacy and security concerns expressed during the beta-test, and to address evolving standards around privacy and security for mobile health apps. Firstly, a 4-digit pin-based 
user login was added as shown in Figure 4. Secondly, a feature which allows the user to login training app only if the phone security lock is enabled. Finally, a feature which invokes user to re-login if the user wants to resume the training app after switching out of it was implemented. To comply with new GDPR privacy guideline, we have included a Privacy policy in the home screen of a training app (Figure 4). The privacy policy was created based on the GDPR template.

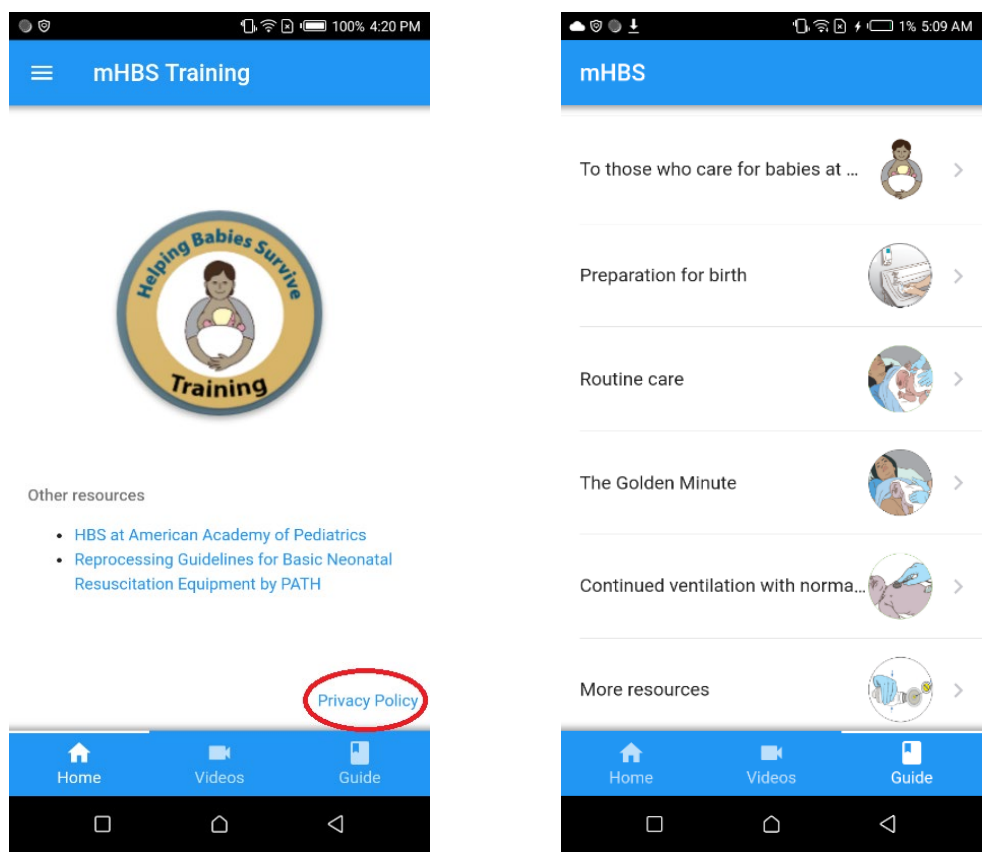

Fig. 5. mHBS training home screen and the HBB guide

\subsection{User interface improvement:}

We received important recommendations to improve stability features from beta-testers. Currently, the training app is available only on Android, even though we use Cordova to build the app. The PDF guide in the training app was digitized into HTML. The guide was divided into eight sections based on the neonatal resuscitation training phases, each section containing respective pages (Fig.5). This modification helps in better navigation control with feasible use of the guide. It also provided us with the ability to capture usability metrics which consequently would be used to evaluate user behavior.

In order to provide HCPs with additional flexibility in viewing, and the ability to resize images in the HTML guide, a zoom function was programmed using the inbuilt class in framework7 called swiper-zoom-container (Figure 6).

\subsection{App usage metrics:}

We utilize Crashlytics as a platform to capture app usage, even though its most intended use is to detect bugs, and app crashes. We created Crashlytics Events to address a variety of questions, including: (1) At what point does an HCP exit a video? (2) How often does an HCP re-watch particular videos? (3) How many times and what pages of a PDF have been viewed? (4) What types of trainer app content have been viewed most frequently and over what period?

These activities are tracked for each user so that the team obtains critical mHBS training app usage information regarding how about, for example, how the healthcare providers progress through self-directed learning modules, and which resources they interact with most frequently, and most thoroughly. Collection of these metrics through the mHBS training app will facilitate future eLearning research which analyzes HCPs behavior patterns for different types of training materials, i.e., video, HTML guide, etc., and how these patterns might influence subsequent knowledge, skills, and competencies, among frontline health workers in LMICs, for maternal and newborn care. 


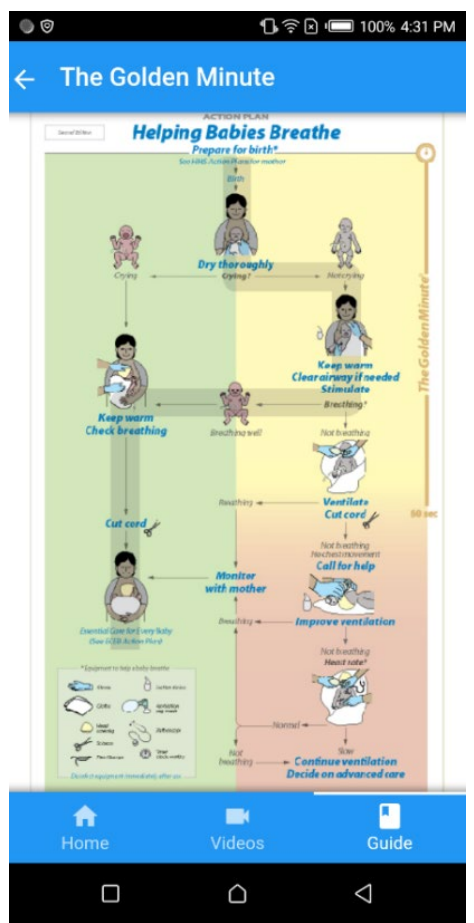

Fig. 6. Zoom functionality

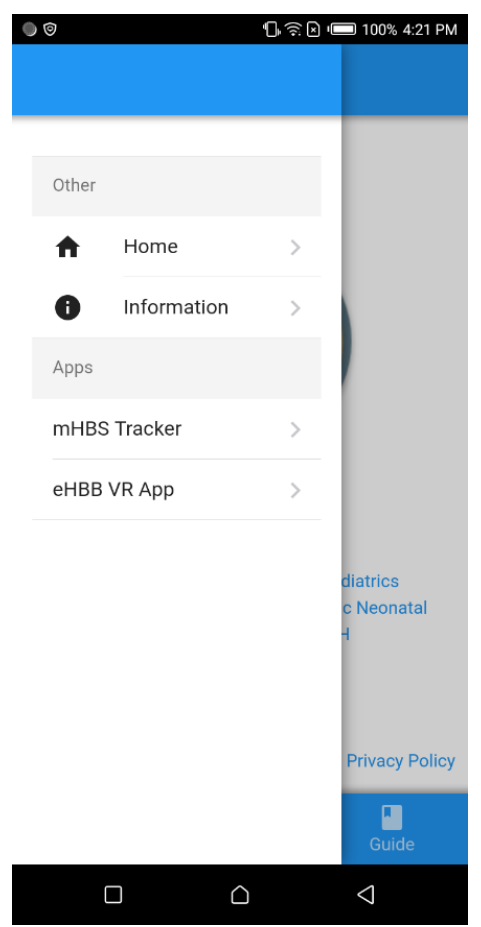

Fig. 7. mHBS training app linking to the mHBS tracker and eHBB app.

\section{Discussion}

\subsection{Insights}

Although not formally analyzed, anecdotally, conducting a comprehensive literature review, and consulting with domain experts prior, to programming and design of the app(s), seemed to assist the technology team in more rapid and effective project progression, and appeared to strengthen interdisciplinary team cohesion among faculty and students. Obtaining an extensive understanding of the myriad non-technical challenges faced by healthcare providers in LMICs lent to more informed technical design. For instance, an HCP employed in a low- supply Neonatal Care Intensive Unit operating at full capacity might need to access an educational module when a birth is occurring. Meanwhile, another user who is currently employed at a higher equipped, low burden hospital may wish to merely review PDFs to supplement their formal training while at home. Understanding the uses and nuances of nurse/midwife-mobile app interaction in low- and middle-income countries (LMICs), such as brownouts that affect Wi$\mathrm{Fi}$, socio-culturally induced stress, overwork and lack of supplies, and availability of mobile charging stations allowed for design considerations that minimized app size, scrolling complexity, and battery usage.

\subsection{Considerations}

Video and documents downloaded in the application are accessed from outside via gallery and file manager in standard Android devices. This does not allow the tracking of this particular type of content usage as accessed by the user. Discussion led to the decision to disable this feature.

\subsection{Limitations}

There are a few limitations which should be mentioned. First, the mHBS application suite is currently built on the assumption that healthcare providers are literate, and have reasonable proficiency, in the English language. Future plans include development of trainer and tracker materials in other languages. Modifications of mHBS/DHIS2 for community-based health care workers with lower levels of literacy may require an increase in the graphical interface, and a decrease in the text. Second, although video thumbnails were successfully gathered and displayed on the training app during one iteration of development, the execution time to generate thumbnails proved to be highly problematic for the user experience, and thus non-viable.

Finally, a lower quantity of screen-by-screen beta-tester feedback was 
acquired than desired. This may have been due to scheduling issues, and the fact that we actively recruited a variety of beta-testers from LMICs - however, due to their extremely busy clinical schedules, and locations across time zones, they were unable to deliver the substantive feedback we requested within a relatively short time period. As a result, most of the feedback received was from highlevel global health and international clinical training experts. This may limit the generalizability of the recommendations regarding utility for frontline health workers. We are currently addressing this concern via an extensive, on-going field test of the mHBS/DHIS2 app among African healthcare providers

\section{Conclusion}

To the best of our knowledge, this is the first time DHIS2 has been used to support video and PDF content for an Android-based mobile app. Our app has improved potential for global scaling and sustainability through the integration with DHIS2. One exciting innovation of our work is that we are employing DHIS2 to track individual health care providers; in the past, DHIS2 has been primarily used for tracking and measuring program or facility-based outcomes. This paper primarily describes the mHBS/DHIS2 Trainer app. As a linked tool, the full mHBS suite of applications (Trainer, Tracker, and Aggregate) provides users with access to integrated functionalities that support self-directed learning, seamless access to external partner resources, and data collection, reporting, and visualization features. This in turn, allows HCPs in LMICs to access a wide variety of high-quality learning materials and training content, for which access metrics are captured. This may inform future research efforts, and lead to improved, more efficient acquisition and retention of knowledge, skills, and competencies for newborn care, thereby leading to a reduction in neonatal mortality in low/middle-income countries.

\section{Acknowledgment}

We would like to acknowledge the contributions of previous developers of the mHBS/DHIS2 application, in particular, Siva Addepally, Olakunle Oladiran, and Taylor Childers. The work reported in this paper was completed as a partial requirement for an undergraduate Capstone Honors Thesis (Informatics) by Ms. Elisabeth Meyers (Drs. Saptarshi Purkayastha and Sherri Bucher, co-Mentors). Ms. Meyers was awarded "Best Scientific Presentation" for this work by the IUPUI Center for Research and Learning on April 6, 2018. We also acknowledge the support of collaborators from University of Washington, Oxford University, Moi University (Kenya) and University of Lagos (Nigeria), as well as the Bill and Melinda Gates Foundation Funding for this work was provided by two grants from the IUPUI Center for Research and Learning, Multidisciplinary Undergraduate Research Initiative (MURI), to Dr. Sherri Bucher (Summer, 2017; Academic year 2017-2018).

\section{References}

1. "Children: reducing mortality", World Health Organization, 2018, http://www.who.int/mediacentre/factsheets/fs178/en/, Last accessed $20 \mathrm{Apr} 2018$

2 J. Pathirana, F. M. Muñoz, V. Abbing-Karahagopian, N. Bhat, T. Harris, A. Kapoor, D. L. Keene, A. Mangili, M. A. Padula, S. L. Pande, V. Pool, F. Pourmalek, F. Varricchio, S. Kochhar, and C. L. Cutland, "Neonatal death: Case definition \& guidelines for data collection, analysis, and presentation of immunization safety data," Vaccine, vol. 34, no. 49, pp. 6027-6037 (2016)

3. H. Ersdal, E. Mduma, E. Svensen and J. Perlman, "Early initiation of basic resuscitation interventions including face mask ventilation may reduce birth asphyxia related mortality in low-income countries", Resuscitation, vol. 83, no. 7, pp. 869-873 (2012)

4. Hoban, R., Bucher, S., Newman, I., Chen, M., Tesfaye, N., Spector, JM. "Helping Babies Breathe" training in sub-Saharan Africa: Educational impact and Learner impressions. J. Trop. Pediatrics, 2013, 59(3), pp. 180-186.

5. Belllad, RM., Bang, A., Carlo WA., McClure EM., Meleth, S., Goco, N., Goudar, SS., Derman RJ., Hibberd PL., Patel A., Esamai F., Bucher, S., Gisore, P., Wright LL., HBB Study Group. "A pre-post study of a multi-country scale up of resuscitation training of facility birth attendants: Does Helping Babies Breathe training save lives? BMC Pregnancy and Childbirth, 2016, August 16(1):222 doi: 10.1186/s12884-016-0997-6.

6. Abwao, S., Bucher, S., Kaimenyi, P., Wachira, J., Esamai, F., Wamae, A. "Kenya Case 
Study" in: Helping Babies Breathe: Lessons learned guiding the way forward. A 5-year report from the HBB Global Development Alliance, June 8, 2015, pp. 99-102.

7. D. Mabey, K. Sollis, H. Kelly, A. Benzaken, E. Bitarakwate, J. Changalucha, X. Chen, Y. Yin, P. Garcia, S. Strasser, N. Chintu, T. Pang, F. Terris-Prestholt, S. Sweeney and R. Peeling, "Point-of-Care Tests to Strengthen Health Systems and Save Newborn Lives: The Case of Syphilis", PLoS Medicine, vol. 9, no. 6, p. e1001233 (2012)

8. D. Tilahun, C. Hanlon, M. Araya, B. Davey, R. Hoekstra and A. Fekadu, "Training needs and perspectives of healthcare providers in relation to integrating child mental health care into primary health care in a rural setting in sub-Saharan Africa: a mixed methods study", International Journal of Mental Health Systems, vol. 11, no. 1, p. 9 (2017)

9. Mobile Applications for the Health Sector, 1st ed. Washington, DC: World Bank, 2018, p. 85

10. I. Medhi, S. Gautama and K. Toyama, "A comparison of mobile money-transfer UIs for non-literate and semi-literate users", Proceedings of the 27th international conference on Human factors in computing systems - CHI 09, p.1742 (2009).

11. Mukherjee, Arunima, Saptarshi Purkayastha, and S. Sahay. "Exploring the potential and challenges of using mobile based technology in strengthening health information systems: Experiences from a pilot study." In AMCIS, p. 263. 2010.

12. C. Hall, E. Fottrell, S. Wilkinson and P. Byass, "Assessing the impact of mHealth interventions in low- and middle-income countries - what has been shown to work?", Global Health Action, vol. 7, no. 1, p. 25606 (2014)

13. "Collect, Manage, Visualize and Explore your Data", Dhis2.org, 2018, https://www.dhis2.org/, Last accessed 21 Apr 2018

14. S. Purkayastha and J. Braa, "Big Data Analytics for developing countries - Using the Cloud for Operational BI in Health," The Electronic Journal of Information Systems in Developing Countries, vol. 59, no. 0, p. 7 (2013)

15. "Ending newborn deaths, ensuring every baby survives", Save the Children, London, 2012. p. 48.

16. Waugaman, Adele. "From principle to practice: implementing the principles for digital development." Proceedings of the Principles for Digital Development Working Group (2016).

17. Bucher SL. mHBB: Using mobile phones to support Helping Babies Breathe in Kenya. In: Levine R, Corbacio A, Konopka S, Saya U, Gilmartin C, Paradis J, Haas S, eds. mHealth Compendium, Volume Five. Arlington, VA: African Strategies for Health, Management Sciences for Health; 2015: 46-47.

18. K. Braa and S. Purkayastha, "Sustainable mobile information infrastructures in low resource settings.," Studies in health technology and informatics, vol. 157, p. 127 (2010)

19. T. Oluoch and N. de Keizer, "Evaluation of Health IT in Low-Income Countries.", IOS Press, vol. 222, p. 324 (2018)

20. "Balsamiq.Rapid, effective and fun wireframing software. Balsamiq", Balsamiq.com, 2018, https://balsamiq.com/, Last accessed 21 Apr 2018.

21. "Free mobile \& web prototyping (iOS, Android) for designers - Marvel", Marvel Prototyping, 2018, https://marvelapp.com/, Last accessed 21 Apr 2018.

22. Lund, Stine, Ida Marie Boas, Tariku Bedesa, Wondewossen Fekede, Henriette Svarre Nielsen, and Bjarke Lund Sørensen. "Association between the safe delivery app and quality of care and perinatal survival in Ethiopia: a randomized clinical trial." JAMA pediatrics 170, no. 8 (2016): 765-771.

23. Harris, Paul A., Robert Taylor, Robert Thielke, Jonathon Payne, Nathaniel Gonzalez, and Jose G. Conde. "Research electronic data capture (REDCap) - a metadata-driven methodology and workflow process for providing translational research informatics support." Journal of biomedical informatics 42, no. 2 (2009): 377-381.

24. "Broadcasts overview. Android Developers", Android Developers, 2018, https://developer.android.com/guide/components/broadcasts, Last accessed 27 Apr 2018

25. "XmlPullParser Android Developers", Android Developers, 2018, https://developer.android.com/reference/org/xmlpull/v1/XmlPullParser, $\quad$ Last accessed 27 Apr 2018

26. "MediaMetadataRetriever. Android Developers", Android Developers, 2018, https://developer.android.com/reference/android/media/MediaMetadataRetriever.h tml, Last accessed 27 Apr 2018

27. "DownloadManager. Android Developers", Android Developers, 2018, https://developer.android.com/reference/android/app/DownloadManager, Last accessed 27 Apr 2018

28. J. Brooke, "SUS-A quick and dirty usability scale," Usability evaluation in industry, vol. 189, no. 194, pp. 4-7 (1996) 